\title{
Fabry Disease: Twenty Novel $\alpha$-Galactosidase A Mutations and Genotype-Phenotype Correlations in Classical and Variant Phenotypes
}

\author{
Dominique P. Germain, ${ }^{1}$ Junaid Shabbeer, ${ }^{2}$ Sylvie Cotigny, ${ }^{1}$ and Robert J. Desnick ${ }^{2}$ \\ ${ }^{1}$ Department of Genetics, Hôpital Européen Georges Pompidou, Paris, France \\ ${ }^{2}$ Department of Human Genetics, Mount Sinai School of Medicine of New York University, New York, \\ New York, USA \\ Contributed by: R. Desnick. Accepted May 15, 2002
}

\begin{abstract}
Background: Fabry disease (OMIM 301500) is an $\mathrm{X}$-linked inborn error of glycosphingolipid metabolism resulting from mutations in the $\alpha$-galactosidase A ( $\alpha$-Gal A) gene. The disease is phenotypically heterogeneous with classic and variant phenotypes. To assess the molecular heterogeneity, define genotype/phenotype correlations, and for precise carrier identification, the nature of the molecular lesions in the $\alpha$-Gal A gene was determined in 40 unrelated families with Fabry disease.

Materials and Methods: Genomic DNA was isolated from affected males or obligate carrier females and the entire $\alpha$-Gal A coding region and flanking sequences were amplified by PCR and analyzed by automated sequencing. Haplotype analyses were performed with polymorphisms within and flanking the $\alpha$-Gal A gene.

Results: Twenty new mutations were identified (G43R, R49G, M72I, G138E, W236X, L243F, W245X, S247C, D266E, W287C, S297C, N355K, E358G, P409S, g1237del15,
\end{abstract}

g10274insG, g10679insG, g10702delA, g11018insA, g11185delT), each in a single family. In the remaining 20 Fabry families, 18 previously reported mutations were detected (R49P, D92N, C94Y, R112C [two families], F113S, W162X, G183D, R220X, R227X, R227Q, Q250X, R301X, R301Q, G328R, R342Q, E358K, P409A, g10208delAA [two families]). Haplotype analyses indicated that the families with the R112C or g10208delAA mutations were not related. The proband with the D266E lesion had a severe classic phenotype, having developed renal failure at 15 years. In contrast, the patient with the S247C mutation had a variant phenotype, lacking the classic manifestations and having mild renal involvement at 64 years.

Conclusions: These results further define the heterogeneity of $\alpha$-Gal A mutations causing Fabry disease, permit precise heterozygote detection and prenatal diagnosis in these families, and provide additional genotype/phenotype correlations in this lysosomal storage disease.

\section{Introduction}

Fabry disease (MIM 301500) is an X-linked recessive inborn error of glycosphingolipid metabolism due to the deficient activity of the lysosomal enzyme, $\alpha$-galactosidase A $(\alpha$ - $D$-galactoside galactohydrolase, EC 3.2.1.22; $\alpha$-Gal A) $(1,2)$. The enzymatic defect results in the accumulation of neutral glycosphingolipids with terminal $\alpha$-galactosyl moieties, particularly globotriaosylceramide (GL-3) (3). Although the glycosphingolipid deposition occurs systemically, the major disease manifestations in classically affected males, who have absent or nondetectable levels of $\alpha$-Gal A activity, primarily result from the progressive accumulation in the microvascular endothelium, leading to ischemia and vascular occlusions. The resultant clinical manifestations include acroparesthesias, which usually begin early in childhood, angiokeratoma, hypohidrosis, and the characteristic corneal and lenticular

Correspondence and reprint requests should be addressed to: Dominique P. Germain, MD, PhD, Department of Genetics, Hôpital Européen Georges Pompidou, 20, rue Leblanc, 75015 Paris, France. Phone: 331560923 06; fax: 331560924 80; e-mail: dominique.germain@hop.egp.ap-hop-paris.fr. opacities. With advancing age, progressive glycosphingolipid deposition in the microvasculature leads to renal failure, cardiac involvement, and cerebrovascular disease.

In classically affected families, heterozygous females for this X-linked disease may have a range of manifestations from asymptomatic to the full blown disease, due to random X-chromosomal inactivation. Most carriers are asymptomatic and live a normal lifespan. Although many carriers have the corneal opacity $(\sim 80 \%)$ that does not affect vision, some have significant cutaneous involvement or report the occurrence of acroparesthesias in childhood or adolescence. Rare carriers with very low or no detectable $\alpha$-Gal A activity have been reported with manifestations as severe as affected males, including the development of renal failure (3).

In contrast, male variants with residual $\alpha$-Gal A activity $(\sim 1-5 \%$ of normal) also have been described (4-7). These males present later in life and lack the classic manifestations of angiokeratoma, acroparesthesias, hypohidrosis, and the characteristic ophthalmologic findings. Among these mildly affected males are the "cardiac variants" who lack the vascular endothelial pathology (4-7) and present in the 
fifth or sixth decade of life with cardiac involvement including left ventricular hypertrophy, cardiomegaly, and conduction abnormalities. They may have proteinuria, but usually do not develop renal failure.

Until recently, the medical management of affected males or symptomatic females with the classic phenotype has consisted of prophylaxis for the acroparesthesias with carbamazepine, diphenylhydantoin, and gabapentin; antihypertensive drugs; and dialysis or renal transplantation for patients experiencing end-stage renal failure. However, enzyme replacement therapy has been shown in recent clinical trials to reverse the disease pathology and markedly improve the well-being and quality of life for classically affected Fabry patients (8-10).

Although the diagnosis of affected males with the classic or variant phenotype can be made reliably by demonstrating the markedly deficient $\alpha$-Gal A activity in plasma, leukocytes, or cultured cells, the enzymatic identification of heterozygous females is less reliable because of random $\mathrm{X}$-chromosome inactivation $(11,12)$. In fact, the finding of normal $\alpha$-Gal A activity in an at-risk female does not exclude heterozygosity, and only the presence of an $\alpha$-Gal A mutation provides precise carrier identification. Thus, molecular testing is required for accurate carrier detection, appropriate genetic counseling, and prenatal diagnosis in affected families.

The isolation and sequencing of the full-length cDNA and entire $\sim 12 \mathrm{~kb}$ genomic sequence encoding $\alpha$-Gal A $(13,14)$ (Genbank X14448) has facilitated characterization of the mutations causing Fabry disease. Of the mutations described to date (Human Gene Mutation Database http://archive.uwcm.ac.uk/ uwcm/mg/hgmd0.html), most have been unique to each family or "private," with the exception of a few mutations found in several unrelated individuals that occurred at $\mathrm{CpG}$ dinucleotides, known hotspots for mutation $(15,16)$.

To investigate the molecular heterogeneity of mutations causing Fabry disease, and to delineate possible phenotype-genotype correlations, mutation analysis of the $\alpha$-Gal A gene was performed in 40 unrelated Fabry families. In 20 families novel mutations were identified; 18 previously reported mutations were detected in the other families. Patients with early-onset classical disease or a mild variant phenotype were identified, thereby providing additional genotype-phenotype information.

\section{Materials and Methods Patient Specimens}

Thirty-eight affected males and two obligate heterozygotes (probands 4 and 38 ) from 40 unrelated families with Fabry disease were evaluated. EDTAanticoagulated whole-blood samples were obtained with informed consent. For each affected male, the enzymatic diagnosis was established by demonstration of deficient $\alpha$-Gal A activity in plasma and/or peripheral leukocytes.

\section{Mutation Analysis}

High-molecular-weight genomic DNA was isolated from leukocytes according to standard procedures. The $\alpha$-Gal A gene, which consists of seven exons distributed over $\sim 12,500 \mathrm{bp}$, was PCR- amplified in four amplicons (17) using the oligonucleotide primers listed in Table 1. The amplicons were purified using exonuclease I and shrimp alkaline phosphatase on a RoboAmp 4200 (MWG Biotech, Courtabeuf, France), and each was sequenced with an ABI Prism 3700 Capillary Array Sequencer using the ABI Prism BigDye Terminator Ready Reaction Mix (Perkin-ElmerCetus, Norwalk, CT, USA). DNA sequences were analyzed using Navigator 2.0 software (PE Biosystems, Norwalk, CT, USA).

\section{Computer Analysis}

Each of the missense lesions was analyzed to determine the relative conservation of the substituted amino acid by comparison with $42 \alpha$-Gal A orthologs (27 eukaryotic and 12 prokaryotic) and five $\alpha$-Gal B orthologs in the GenBank database (http://www.ncbi.nih.gov/Entrez/nucleotide.html).

Table 1. Oligonucleotide primers used for PCR amplification and sequencing of the $\alpha$-Gal A gene

\begin{tabular}{clllr}
\hline Amplicon & Exon(s) Amplified & Primer Sequence $\left(5^{\prime} \rightarrow \mathbf{3}^{\prime}\right)$ & Orientation & Genomic Sequence* $^{*}$ \\
\hline \multirow{2}{*}{1} & Promoter and exon 1 & AAGCACGCATTTGCCTAGAT & Forward & $330-349$ \\
& & TTTAGGGCTAGTCCTGATTC & Reverse & $1526-1545$ \\
2 & Exon 2 & GCATTTTAGGTTGTTCAGTTG & Forward & $4715-4735$ \\
& & ACAGAAGTGCTTACAGTCCTC & Reverse & $5301-5321$ \\
3 & Exons 3 and 4 & CCTAGTTTGAACTAGCTCAG & Forward & $7059-7078$ \\
& & GACAGGACATGAATGGTGAA & Reverse & $8623-8642$ \\
4 & Exons $5-7$ & CTTTAAGGGATTTCAAGTTA & Forward & $9930-9949$ \\
& & TTGAGATGGAATCTTTGTTG & Reverse & $11588-11607$
\end{tabular}

${ }^{*} \alpha$-Gal A genomic residues numbered according to Kornreich et al. (14). 
These searches were performed using the MacVector program (Oxford Molecular Group). Highly conserved residues were defined as those that were present in all three mammalian orthologs, in at least 20 of 27 eukaryotic orthologs, and in 4 of $5 \alpha$-Gal B orthologs, except for the lesions that occurred in $\alpha$-Gal A exon 7 , which has little (16\%), if any, amino acid identity with the corresponding region of $\alpha$-Gal B (18).

\section{Results}

To identify the $\alpha$-Gal A mutations in 40 unrelated families with Fabry disease, four amplicons containing the seven $\alpha$-Gal A exons and their adjacent flanking and intronic regions were PCR-amplified and sequenced. No size abnormalities were detected when the amplicons were analyzed by agarose gel electrophoresis. In contrast, direct automated sequencing of each amplicon detected a single mutation in each Fabry proband, including 20 novel and 18 previously reported mutations (Table 2 ). The 20 novel mutations included 12 missense and 2 nonsense mutations, 3 small deletions, and 3 small insertions as described below. The previously reported lesions included 12 missense mutations-R49P, D92N, C94Y, R112C (two families), F113S, G183D, R227Q，R301Q，G328R， R342Q，E358K, and P409A-5 nonsense mutations-W162X, R220X, R227X, Q250X, and R301X-and I small deletion g10208delAA (two families) (Fig. 1).

As indicated in Table 2, the novel missense mutations included:

1. a G-to-C transversion of genomic nucleotide (g) 1306 in codon 43 of exon 1 ( $\underline{G G C} \rightarrow \underline{C G C}$ ), replacing a neutral, polar highly conserved (Table 3 ) glycine with a basic arginine (G43R);
2. a C-to-G transversion of g1324 in codon 49 of exon 1 ( $\underline{G} G C \rightarrow \underline{G G C})$, replacing a basic arginine with a neutral, polar glycine (R49G);

3. a G-to-A transition of g5115 in codon 72 of exon 2 (AT $\underline{G} \rightarrow$ AT $\underline{A})$, replacing a neutral, polar methionine with an isoleucine (M72I);

4. a G-to-A transition of g7312 in codon 138 of exon 3 (GGA $\rightarrow$ GAA), substituting the neutral, polar, highly conserved glycine with a glutamic acid (G138E);

5. a G-to-C transversion of g10220 in codon 243 of exon 5 (TT $\underline{G} \rightarrow$ TTC $)$, changing a leucine to a phenylalanine (L243F);

6. a C-to-G transversion of g10231 in codon 247 of exon $5(\mathrm{~T} \underline{\mathrm{T}} \rightarrow \mathrm{T} \underline{\mathrm{G}} \mathrm{T})$, resulting in the replacement of a serine by a cysteine (S247C);

7. a T-to-A transversion of g 10289 in codon 266 of exon 5 (GAT $\rightarrow$ GAA), resulting in the substitution of glutamic acid for a highly conserved aspartic acid (D266E);

8. a G-to-T transversion of g10569 in codon 287 of exon 6 (TGG $\rightarrow T G \underline{T})$, substituting a cysteine for tryptophan (W287C);

9. a C-to-G transversion of g10598 in codon 297 of exon $6(\mathrm{~T} \underline{\mathrm{C}} \rightarrow \mathrm{T} \underline{\mathrm{GT}})$, substituting a serine by a cysteine (S297C);

10. a C-to-A transversion of g1 1043 in codon 355 of exon $7(\mathrm{AAC} \rightarrow \mathrm{AAA})$, resulting in the replacement of a neutral, nonpolar asparagine with a neutral, polar lysine (N355K);

11. an A-to-G transition of g11051 in codon 358 of exon 7 (GAG $\rightarrow G \underline{G G})$, replacing a glutamic acid with a glycine (E358G). This mutation differs from the already described E358K (GAG $\rightarrow$ AAG) allele, which occurs in the same codon (19); and

12. a C-to-T transition of gl1203 in codon 409 of exon $7(\underline{C} C \rightarrow \underline{T C C})$, replacing a neutral, polar

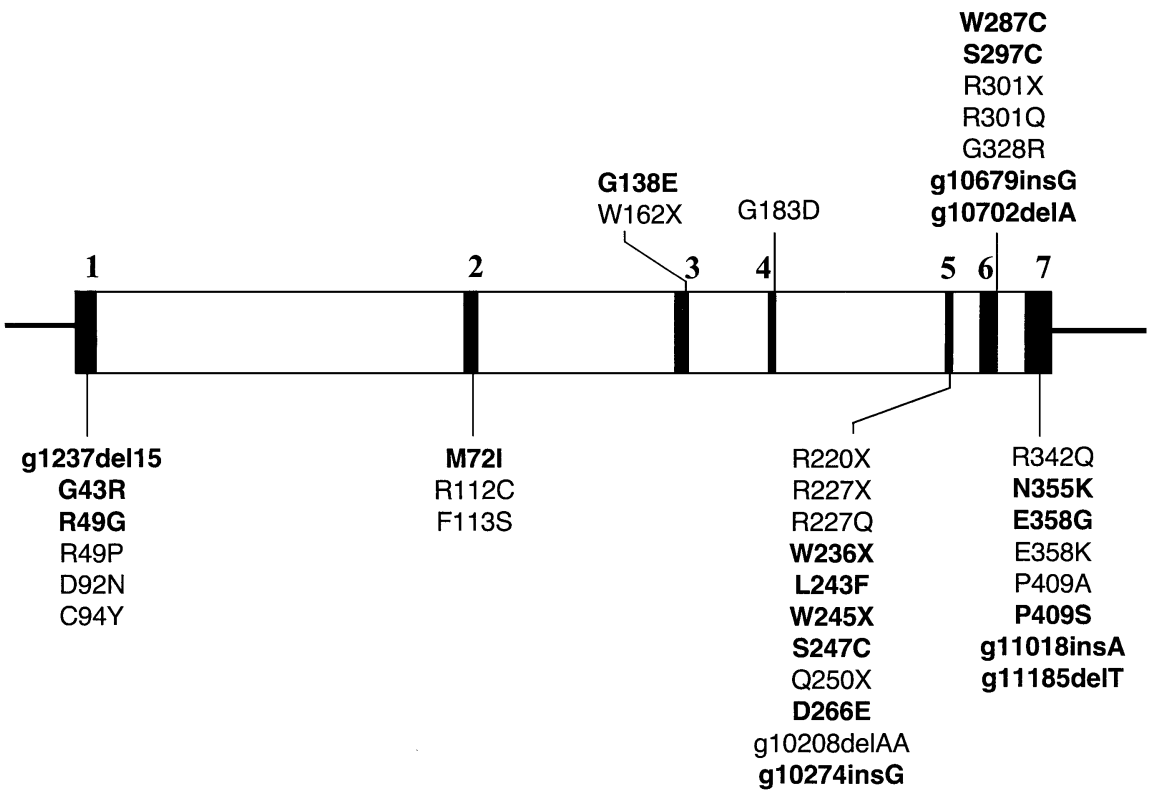

Fig. 1. Distribution of the 20 novel and 18 previously reported mutations in the $\alpha$-Gal A gene identified in this study. Exons are indicated as solid rectangles and the novel mutations are indicated in bold. 
Table 2. $\alpha$-Gal A mutations causing Fabry disease in 40 unrelated families

\begin{tabular}{|c|c|c|c|c|c|c|c|}
\hline Proband & Mutation & Location & $\begin{array}{c}\text { Genomic } \\
\text { Nucleotide Change }\end{array}$ & $\begin{array}{c}\text { cDNA } \\
\text { Codon Change }\end{array}$ & $\begin{array}{l}\text { Age } \\
\text { (Yr) }\end{array}$ & Origin & Phenotype \\
\hline \multicolumn{8}{|c|}{ Novel mutations } \\
\hline \multicolumn{8}{|c|}{ Missense mutations } \\
\hline 1 & G43R & Exon 1 & g1306 G > C & $127 \mathrm{GGC}>\mathrm{CGC}$ & 22 & British & Classic \\
\hline 2 & $\mathrm{R} 49 \mathrm{G}$ & Exon 1 & g1324 C > G & $145 \mathrm{CGC}>\mathrm{GGC}$ & 57 & French & Classic \\
\hline 3 & M72I & Exon 2 & g5115 G > A & 216 ATG $>$ ATA & 61 & French & Classic \\
\hline 4 & G138E & Exon 3 & g7312 G > A & 413 GGA $>$ GAA & 56 & German & Classic \\
\hline 5 & L243F & Exon 5 & gl0220 G > C & 729 TTG $>$ TTC & 37 & French & Classic \\
\hline 6 & S247C & Exon 5 & g10231 C > G & 740 TCT $>$ TGT & 64 & French & Variant \\
\hline 7 & D266E & Exon 5 & g10289 T > A & 798 GAT $>$ GAA & 31 & French & Severe classic \\
\hline 8 & W287C & Exon 6 & g10569 G > T & $861 \mathrm{TGG}>\mathrm{TGT}$ & 29 & British & Classic \\
\hline 9 & S297C & Exon 6 & gl0598 C > G & $890 \mathrm{TCT}>\mathrm{TGT}$ & 20 & Belgian & Classic \\
\hline 10 & N355K & Exon 7 & g11043 C > A & $1065 \mathrm{AAC}>\mathrm{AAA}$ & 16 & French & Classic \\
\hline 11 & E358G & Exon 7 & g11051 A > G & $1073 \mathrm{GAG}>\mathrm{GGG}$ & 41 & West Indian & Classic \\
\hline 12 & P409S & Exon 7 & g11203 C > T & 1225 CCC $>$ TCC & 38 & British & Classic \\
\hline \multicolumn{8}{|c|}{ Nonsense mutations } \\
\hline 13 & W236X & Exon 5 & g10198 G > A & $707 \mathrm{TGG}>\mathrm{TAG}$ & 32 & British & Classic \\
\hline 14 & W245X & Exon 5 & g10225 G > A & 734 TGG $>$ TAG & 50 & French & Classic \\
\hline \multicolumn{8}{|c|}{ Small deletions } \\
\hline 15 & 58del15 & Exon 1 & $\begin{array}{l}\text { g1237delGCCCTCG } \\
\text { TTTCCTGG }\end{array}$ & $\begin{array}{l}\text { 58delGCCCTCG } \\
\text { TTTCCTGG }\end{array}$ & 40 & Kurdish & Classic \\
\hline 16 & CTT $\underline{A}$ GA CAG* & Exon 6 & g10702delA & 994delA & 19 & Iraqi & Classic \\
\hline 17 & AGG TTA AGA & Exon 7 & g11185delT & 1207 delT & 28 & French & Classic \\
\hline \multicolumn{8}{|c|}{ Small insertions } \\
\hline 18 & CCA GGG GGG & Exon 5 & g10274insG & 778insG & 29 & French & Classic \\
\hline 19 & TTG G G G CAA & Exon 6 & g10679insG & 972insG & 21 & French & Classic \\
\hline 20 & TTA $\underline{A} G C$ CTG & Exon 7 & g11018insA & 104linsA & 50 & British & Classic \\
\hline \multicolumn{8}{|c|}{ Previously reported mutations } \\
\hline \multicolumn{8}{|c|}{ Missense mutations } \\
\hline 21 & $\mathrm{R} 49 \mathrm{P}$ & Exon 1 & g1325 G > C & $146 \mathrm{CGC}>\mathrm{CCC}$ & 24 & British & Classic \\
\hline 22 & D92N & Exon 1 & g5176 G > A & $274 \mathrm{GAT}>\mathrm{AAT}$ & 30 & British & Classic \\
\hline 23 & $\mathrm{C} 94 \mathrm{Y}$ & Exon 1 & g5180 G > C & $281 \mathrm{TGT}>\mathrm{TCT}$ & 13 & Hispanic & Classic \\
\hline 24 & $\mathrm{R} 112 \mathrm{C}$ & Exon 2 & g5233 C > T & $334 \mathrm{CGC}>\mathrm{TGC}$ & 33 & French & Classic \\
\hline 25 & R112C & Exon 2 & g5233 C > T & $334 \mathrm{CGC}>\mathrm{TGC}$ & 47 & French & Classic \\
\hline 26 & F113S & Exon 2 & g5237 T > C & 338 TTT $>$ TCT & 47 & British & Classic \\
\hline 27 & G183D & Exon 4 & g8321 G > A & $548 \mathrm{GGT}>\mathrm{GAT}$ & 31 & French & Classic \\
\hline 28 & $\mathrm{R} 227 \mathrm{Q}$ & Exon 5 & $\mathrm{~g} 10171 \mathrm{G}>\mathrm{A}$ & $680 \mathrm{CGA}>\mathrm{CAA}$ & 39 & British & Classic \\
\hline 29 & R301Q & Exon 6 & $\mathrm{~g} 10610 \mathrm{G}>\mathrm{A}$ & $902 \mathrm{CGA}>\mathrm{CAA}$ & 60 & French & Intermediate \\
\hline 30 & G328R & Exon 6 & $\mathrm{~g} 10690 \mathrm{G}>\mathrm{A}$ & $982 \mathrm{GGG}>\mathrm{AGG}$ & 26 & Arab & Classic \\
\hline 31 & $\mathrm{R} 342 \mathrm{Q}$ & Exon 7 & $\mathrm{~g} 11003 \mathrm{G}>\mathrm{A}$ & $1025 \mathrm{CGA}>\mathrm{CAA}$ & 33 & French & Classic \\
\hline 32 & E358K & Exon 7 & g11050 G $>A$ & $1072 \mathrm{GAG}>\mathrm{AAG}$ & 39 & French & Classic \\
\hline 33 & P409A & Exon 7 & g11203 C > G & $1225 \mathrm{CCC}>\mathrm{GCC}$ & 29 & British & Classic \\
\hline \multicolumn{8}{|c|}{ Nonsense mutations } \\
\hline 34 & W162X & Exon 3 & g7384 G > A & $485 \mathrm{TGG}>\mathrm{TAG}$ & 39 & British & Classic \\
\hline 35 & R220X & Exon 5 & g10149 C > T & $658 \mathrm{CGA}>\mathrm{TGA}$ & 36 & British & Classic \\
\hline 36 & $\mathrm{R} 227 \mathrm{X}$ & Exon 5 & $\mathrm{~g} 10170 \mathrm{C}>\mathrm{T}$ & $679 \mathrm{CGA}>\mathrm{TGA}$ & 59 & French & Classic \\
\hline 37 & Q250X & Exon 5 & g10239 C > T & $748 \mathrm{CAG}>\mathrm{TAG}$ & 53 & French & Classic \\
\hline 38 & $\mathrm{R} 301 \mathrm{X}$ & Exon 6 & g10609 C > T & $901 \mathrm{CGA}>\mathrm{TGA}$ & 48 & $\begin{array}{l}\text { French } \\
\text { Canadian }\end{array}$ & Classic \\
\hline \multicolumn{8}{|c|}{ Small deletions } \\
\hline 39 & AGT ATA AAG AGT & Exon 5 & g10208delAA & 717delAA & 50 & French & Classic \\
\hline 40 & AGT ATA $\underline{A} A G$ AGT & Exon 5 & g10208delAA & 717delAA & 20 & French & Classic \\
\hline
\end{tabular}

*Inserted or deleted bases underlined.

proline with a neutral, nonpolar serine (P409S).

The relative conservation during evolution of the amino acids substituted by the missense mutations is indicated in Table 3.
The two novel nonsense mutations included:

1. a G-to-A transition of g10198 in codon 236 of exon 5 (T $\underline{G G}$ to TAGG), predicting a termination signal instead of a tryptophan (W236X), and deletion of 194 residues; and 
Table 3. Conservation of human $\alpha$-Gal A missense mutations in $\alpha$-Gal A and $\alpha$-Gal B orthologues

\begin{tabular}{|c|c|c|c|c|c|c|c|}
\hline \multirow[b]{2}{*}{ Proband } & \multirow[b]{2}{*}{ Exon } & \multirow[b]{2}{*}{ Mutation } & \multicolumn{3}{|c|}{$\alpha$-Gal A Orthologs } & \multirow{2}{*}{$\begin{array}{c}\alpha \text {-Gal B Orthologs } \\
\text { Eukaryotic } \\
(5)\end{array}$} & \multirow[b]{2}{*}{ Conservation* } \\
\hline & & & $\begin{array}{l}\text { Prokaryotic } \\
\text { (12) }\end{array}$ & $\begin{array}{c}\text { Eukaryotic } \\
(27)\end{array}$ & $\begin{array}{c}\text { Mammalian } \\
\text { (3) }\end{array}$ & & \\
\hline 1 & 1 & G43R & 5 & 22 & 3 & 5 & +++ \\
\hline 2 & 1 & R49G & 1 & 1 & 3 & 4 & + \\
\hline 3 & 2 & M72I & 0 & 0 & 3 & 4 & + \\
\hline 4 & 3 & G138E & 3 & 24 & 3 & 5 & +++ \\
\hline 5 & 5 & L243F & 0 & 4 & 3 & 3 & - \\
\hline 6 & 5 & S247C & 1 & 3 & 1 & 0 & - \\
\hline 7 & 5 & D266E & 5 & 22 & 3 & 5 & +++ \\
\hline 8 & 6 & W287C & 7 & 25 & 3 & 5 & +++ \\
\hline 9 & 6 & S297C & 2 & 2 & 3 & 5 & + \\
\hline 10 & 7 & N355K & 2 & 23 & 3 & 2 & +++ \\
\hline 11 & 7 & E358G & 3 & 1 & 3 & 1 & - \\
\hline 12 & 7 & P409S & 2 & 6 & 3 & 5 & + \\
\hline
\end{tabular}

*For the degree of evolutionary conservation:-, minimal conservation; +, conserved among all three $\alpha$-Gal A mammalian orthologs and four of five $\alpha$-Gal B eukaryotic orthologues, with the exception of the $\alpha$-Gal A exon 7 mutations (18); ++ , additionally or alternately conserved among 14-23 of $\alpha$-Gal A eukaryotic orthologs; +++, conserved among all three $\alpha$-Gal A mammalian orthologs and five $\alpha$-Gal B eukaryotic orthologs, and at least $22 \alpha$-Gal A eukaryotic orthologues.

2. a G-to-A transition of g10225 in codon 245 of

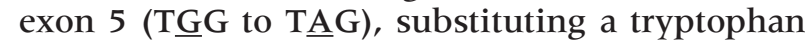
codon by a termination signal (W245X), and deletion of 184 residues.

Six novel small gene rearrangements, including two single nucleotide insertions and three deletions were identified in six unrelated classically affected probands (Table 2). The three small insertions were

1. a single nucleotide insertion after g10273 (gl0273insG) that caused a frameshift after codon 259, altered residues 262 and 263, and then introduced a termination signal at codon 264;

2. a single nucleotide insertion after g10682 (gl0682insG) that caused a frameshift after codon 224, altered residues 326 to 331 , and prematurely terminated the polypeptide after residue 332; and

3. a single nucleotide insertion after g11018 (g11018insA) that caused a frameshift after codon 347, altered residues 348-373, and then introduced a termination signal at codon 374 .

The three novel gene deletions included:

1. an in-frame 15-bp deletion in exon 1 (58del15) that resulted in the deletion of amino acids 20 to 24 (ALVSW) from the 31 residue leader sequence;

2. a single nucleotide deletion (g11185delT) at codon 403, predicting a frameshift and premature termination in that codon; and
3. a single base deletion (994delA) of g10702 that introduced a frameshift after codon 331, altered residues 332-346, and then introduced a termination signal at codon 347.

\section{Discussion}

Sequencing of the $\alpha$-Gal A gene in 40 unrelated families with Fabry disease revealed 20 novel and 18 previously reported mutations. Each of the 20 novel mutations was identified in a single family, demonstrating the extensive molecular heterogeneity in this disease. The novel mutations were dispersed along the gene, and included 12 missense and 2 nonsense point mutations, 3 small insertions, and 3 small deletions, all in the coding region. No novel mutation occurred at a CpG dinucleotide, whereas 7 (R112C, R220X, R227X, R227Q, R301X, R301Q, R342Q) of the 18 previously reported mutations occurred at CpG dinucleotides, known hotspots for mutation. Of the 12 novel missense mutations, only 5 (G43R, G138E, D266E, W287C, and N355K) occurred at highly conserved residues (Table 3 ). Notably, among these mutations was D266E, which substituted a highly conserved aspartate with an isofunctional glutamate. The aspartate must be essential for enzyme activity (or stability) because substitution of the same charged, but slightly longer, glutamate residue caused Fabry disease.

Of the six novel gene rearrangements, exons 6 and 7 each had two. The identification of these small deletions and insertions supports the previous suggestion that exons 6 and 7 are regions prone to small gene rearrangements $(20,21)$. Two of the three small insertions involved the addition of an extra $G$ to a 
small series of Gs ( 5 in 778insG and 3 in 972insG), presumably due to DNA replication errors. The 58 del15 mutation, which eliminated five amino acids in the leader sequence, resulted in an in-frame deletion of $15 \mathrm{bp}$, presumably due to slipped mispairing during replication between 7-bp direct repeats (TTCTGG) such that one direct repeat and the intervening sequence were deleted. The five deleted residues were in the central hydrophobic core of the leader. When evaluated by the von Heijne algorithm (22), loss of these residues altered the most likely leader sequence cleavage site from the normal site after residue 31 to several sites in exon 7 . Thus, the polypeptide may not be targeted to the endoplasmic reticulum, or if it is, it would be miscleaved.

These studies provided additional genotypephenotype information for Fabry disease. Most of the affected males described had the classic phenotype. Although the age of onset and/or severity of the manifestations varied, these patients presented with angiokeratoma, hypohidrosis, and acroparesthesias in childhood, and developed renal insufficiency, cardiac, or cerebrovascular disease in the fourth or fifth decades of life. Notably, several variants were identified. Proband number 7 with the D266E mutation progressed to renal failure early; he began dialysis at the age of 15 years, and at 16 years received a kidney allograft, which has remained functional for 15 years. Only a few other affected males whose genotypes were not reported have experienced early renal failure $(23,24)$. Although the aspartic acid to glutamic acid substitution at residue 266 is isofunctional, the aspartate is highly conserved in evolution, being present among all the higher eukaryotes, the majority (19 of 24) of the lower eukaryotes, and all of the $\alpha$-Gal B orthologs (Table 3). In contrast, the affected male with the S247C mutation had a milder variant phenotype. He did not have the classic disease manifestations (acroparesthesia, angiokeratoma, hypohidrosis, or corneal dystrophy). Instead, he presented at age 64 with mild proteinuria and moderate renal insufficiency (serum creatinine, $1.7 \mathrm{mg} / \mathrm{dl}$; glomerular filtration rate by $\mathrm{EDTA}^{-51} \mathrm{Cr}, 41.2 \mathrm{ml} / \mathrm{min} / 1.73 \mathrm{~m}^{2}$; normal, $63-119 \mathrm{ml} / \mathrm{min} / 1.73 \mathrm{~m}^{2}$ ). He had low, but detectable, plasma and leukocyte $\alpha$-Gal A levels, which were consistent with his mild phenotype. These findings suggested that he has an intermediate phenotype, perhaps similar to the recently described renal variant (Nakao et al., in review). Of note, the serine at residue 247 is not evolutionarily conserved, only being present in the spider monkey, three lower eukaryotes, and the bacterium Thermotoga maritima (Table 3).

Among the previously reported mutations detected in this study, it is interesting to note that the R301Q mutation was initially identified in a patient with the cardiac variant (25). However, the patient reported here developed renal insufficiency and received a kidney transplant at age 59 (6). This mutation apparently produces a small amount of residual activity, and presumably its synthesis and/or stability are subject to variation. A similar clinical course was reported for an unrelated classically affected male (26) and a mildly affected male with an "intermediate" phenotype (8) who had the R301Q mutation and developed renal failure.

Among mutation detection methods, singlestrand conformational polymorphism (27) and chemical cleavage of mismatches (28) have been used effectively to detect $\alpha$-Gal A mutations. However, DNA sequencing remains the "gold standard" for mutation detection. With the improvement in both sequencing chemistries and attendant softwares for mutation detection, direct automated DNA sequencing has now become even more practical for routine mutation detection involving genes with a moderate number of exons, like $\alpha$-Gal A $(20,26,29)$.

In summary, the identification of 20 novel $\alpha$-Gal A mutations increased our understanding of the molecular heterogeneity and the genotype/phenotype correlations in Fabry disease. In addition, these studies permit precise carrier detection, genetic counseling, and prenatal diagnosis in these 40 families with this X-linked lysosomal disease.

\section{Acknowledgments}

We are indebted to the patients, their families, and our nursing staff for their help with this study. D.P.G. was supported by Vaincre les Maladies Lysosomales (VML). This work also was supported in part by grants from the National Institutes of Health including a research grant (R37 DK 34045 Merit Award), a grant (5 MOl RR00071) for the Mount Sinai General Clinical Research Center from the National Center of Research Resources, and a grant (5 P30 HD28822) for the Mount Sinai Child Health Research Center.

\section{References}

1. Brady RO, Gal AE, Bradley RM, Martensson E, Warshaw AL, Laster L. (1967) Enzymatic defect in Fabry's disease. Ceramidetrihexosidase deficiency. N. Engl. J. Med. 276: 1163-1 167.

2. Kint JA. (1970) Fabry's disease: alpha-Galactosidase deficiency. Science 167: 1268-1269.

3. Desnick RJ, Ioannou YA, Eng CM. (2001) $\alpha$-Galactosidase A deficiency: Fabry disease. In: Scriver CR, Beaudet AL, Sly WS, Valle D, Kinzler KE, Vogelstein B (eds). The Metabolic and Molecular Bases of Inherited Disease. 8th ed., New York: McGrawHill; 3733-3774.

4. von Scheidt W, Eng CM, Fitzmaurice TF, et al. (1991) An atypical variant of Fabry's disease with manifestations confined to the myocardium. N. Engl. J. Med. 324: 395-399.

5. Nakao S, Takenaka T, Maeda M, et al. (1995) An atypical variant of Fabry's disease in men with left ventricular hypertrophy. N. Engl. J. Med. 333: 288-293.

6. Germain DP. (2001) A new phenotype of Fabry disease with intermediate severity between the classical form and the cardiac variant. Contrib. Nephrol. 136: 234-240.

7. Elleder M, Bradova V, Smid F, et al. (1990) Cardiocyte storage and hypertrophy as a sole manifestation of Fabry's disease. Report on a case simulating hypertrophic non-obstructive 
cardiomyopathy. Virchows Arch. A Pathol. Anat. Histopathol. 417: 449-455.

8. Eng CM, Banikazemi M, Gordon R, et al. (2001) A phase I/II clinical trial of enzyme replacement in Fabry disease: Pharmacokinetic, substrate clearance, and safety studies. Am. J. Hum. Genet. 68: 711-722.

9. Eng CM, Guffon N, Wilcox WR, et al. (2001) Safety and efficacy of recombinant human $\alpha$-galactosidase A replacement therapy in Fabry's disease. N. Engl. J. Med. 345: 9-16.

10. Schiffmann R, Kopp JB, Austin HA, 3rd, et al. (2001) Enzyme replacement therapy in Fabry disease: a randomized controlled trial. JAMA 285: 2743-2749.

11. Lyon M. (1961) Gene action in the X-chromosome of the mouse (Mus musculus L.). Nature 190: 372-373.

12. Brown RM, Brown GK. (1993) X-chromosome inactivation and the diagnosis of X-linked disease in females. J. Med. Genet. 30: 177-184.

13. Bishop DF, Calhoun DH, Bernstein HS, Hantzopoulos $P$, Quinn M, Desnick RJ. (1986) Human $\alpha$-galactosidase A: Nucleotide sequence of a cDNA clone encoding the mature enzyme. Proc. Natl. Acad. Sci. U.S.A. 83: 4859-4863.

14. Kornreich R, Desnick RJ, Bishop DF. (1989) Nucleotide sequence of the human $\alpha$-galactosidase A gene. Nucl. Acids. Res. 17: 3301-3302.

15. Barker DF, Schafer M, White R. (1984) Restriction sites containing CpG show a higher frequency of polymorphism in human DNA. Cell 36: 131-138.

16. Cooper C, Youssoufian H. (1988) The CpG dinucleotide and human genetic disease. Hum. Genet. 78: 151-155.

17. Kornreich R, Desnick RJ. (1993) Fabry disease: Detection of gene rearrangements in the human $\alpha$-galactosidase A gene by multiplex PCR amplification. Hum. Mutat. 2: 108111 .

18. Wang AM, Desnick RJ. (1991) Structural organization and complete sequence of the human $\alpha$-N-acetylgalactosaminidase gene: homology with the $\alpha$-galactosidase A gene proves evidence for evolution from a common ancestral gene. Genomics 10: 133-142.
19. Miyazaki T, Kajita M, Ohmori S, et al. (1998) A novel mutation $(\mathrm{E} 358 \mathrm{~K})$ in the $\alpha$-galactosidase A gene detected in a Japanese family with Fabry disease. Hum. Mutat. Suppl 1: S139-140.

20. Ashley GA, Shabbeer J, Yasuda M, Eng CM, Desnick RJ. (2001) Fabry disease: twenty novel $\alpha$-galactosidase A mutations causing the classical phenotype. J. Hum. Genet. 46: 192-196.

21. Eng CM, Ashley GA, Burgert TS, Enriquez AL, D'Souza M, Desnick RJ. (1997) Fabry disease: thirty-five mutations in the $\alpha$-galactosidase A gene in patients with classic and variant phenotypes. Mol. Med. 3: 174-182.

22. Nielsen H, Engelbrecht J, Brunak S, von Heijne G. (1997) Identification of prokaryotic and eukaryotic signal peptides and prediction of their cleavage sites. Protein Eng. 10: 1-6.

23. Grunfeld JP, Lidove O, Joly D, Barbey F. (2001) Renal disease in Fabry patients. J. Inherit. Metab. Dis. 24(suppl 2): 71-74.

24. Sheth KJ, Roth DA, Adams MB. (1983) Early renal failure in Fabry's disease. Am. J. Kidney Dis. 2: 65 1-654.

25. Sakuraba H, Oshima A, Fukuhara Y, et al. (1990) Identification of point mutations in the $\alpha$-galactosidase A gene in classical and atypical hemizygotes with Fabry disease. Am. J. Hum. Genet. 47: 784-789.

26. Ashton-Prolla P, Tong B, Astrin $\mathrm{KH}$, Shabbeer J, Eng CM, Desnick RJ. (2000) 22 novel mutations in the $\alpha$-galactosidase A gene and genotype/phenotype correlations including mild hemizygotes and severely affected heterozygotes. J. Invest. Med. 48: 227-235.

27. Blaydon D, Hill JA, Winchester B. (2001) Fabry disease: 20 novel GLA mutations in 35 families. Human Mutation Online 18: 459.

28. Germain D, Biasotto M, Tosi M, Meo T, Kahn A, Poenaru L. (1996) Fluorescence-assisted mismatch analysis (FAMA) for exhaustive screening of the $\alpha$-galactosidase A gene and detection of carriers in Fabry disease. Hum. Genet. 98: 719-726.

29. Topaloglu AK, Ashley GA, Tong B, et al. (1999) Twenty novel mutations in the $\alpha$-galactosidase A gene causing Fabry disease. Mol. Med. 5: 806-811. 